\title{
INTERSTITIAL PLASMACELLULAR PNEUMONIA DUE TO PNEUMOCYSTIS CARINII
}

\author{
BY \\ H. S. BAAR \\ From the Department of Pathology, the Children's Hospital, Birmingham
}

(RECEIVED FOR PUBLICATION MAY 24, 1954)

Interstitial plasmacellular pneumonia of young infants is a fairly common disease on the European continent. It has been described as a new morbid entity by Rössle $(1923,1928)$ and Loeschcke (1928) and in more detail by Benecke (1938), Ammich (1938), Feyrter (1927, 1941), Roulet (1941), Gloggengiesser (1951), and others. Nitschke (1940) published a thorough review of clinical symptoms and radiological findings, and Weisse (1951) recently wrote an extensive monograph. The disease occurs mainly, but not exclusively, in premature infants between 2 and 4 months of age, and almost exclusively in those who have been in hospital for some time. Clinically it is characterized by severe respiratory distress and cyanosis with little or no fever and no definite physical signs over the chest but marked radiological changes. Almost all reports are from Switzerland, Germany, Austria, and Czechoslovakia, only a very few from northern European countries (Linell, 1949 ; Gormsen, 1950), none from southern Europe, and none from outside the European continent.

The purpose of the present paper is to report the first case outside the European continent, to describe the histochemical characteristics of the peculiar honeycombed masses which fill the air spaces, to demonstrate the histopathological differences between plasmacellular interstitial pneumonia and other forms of interstitial pneumonia of infancy, to confirm the finding of Vaněk and Jirovec (1952) of the protozoon Pneumocystis carinii in the air spaces, and to discuss briefly the curious association with cytomegalic disease of salivary glands.

\section{Case Report}

The patient, a girl, was born at full term in an institution in the Midlands with a birth weight of $6 \mathrm{lb}$. She was 12 weeks old at the time of death. The mother suffered each winter from respiratory tract infection. The baby was well until the end of December, 1950, eight days before death, when she developed a nasal discharge. Four days before death she developed a cough which was occasionaliy paroxysmal and associated with vomiting. Next day tachypnoea and cyanosis were noticed. On admission, two days before death, she was distressed and cyanosed. Rhonchi and rales were heard over both lungs. Her temperature was $100^{\circ} \mathrm{F}$., the white cell count 13,200 with $51 \%$ polymorphonuclear leucocytes. The mother had her usual respiratory tract infection in December. There was no history of respiratory tract infections in the maternity home where the baby was born.

\section{Pathology}

At the necropsy the pleurae were normal ; the trachea and the main bronchi contained a small amount of mucopurulent exudate but their mucosa appeared normal. The lungs were bulky, but there was no pus in the smaller bronchi and bronchioli. Scattered throughout all lobes of both lungs were ill-defined, greyish-red areas of consolidation, 2-3 $\mathrm{mm}$. in diameter, and between these there were grey spots and broad grey strands, apparently corresponding to broadened interstitial tissue. The tracheo-bronchial lymph nodes were of normal appearance. The spleen was enlarged $(23 \mathrm{~g}$.). The Malpighian corpuscles were rather conspicuous, the pulp greyish-red and not diffluent. The liver showed moderate fatty changes, the kidneys cloudy swelling, the other organs no abnormality.

Microscopical Examination.-In the lungs all alveolar ducts were distended and, like the less distended alveoli, filled with a peculiar foamy or honeycombed material (Fig. 1).

In some of the air spaces there were also red blood cells which showed little alteration. Alveolar macrophages were seen only in a few alveolar ducts and alveoli. Alveoli, alveolar ducts, and respiratory bronchioles were all lined with epithelial cells which were usually cuboid, sometimes low columnar, and sometimes moderately flattened (Fig. 2). These cells were rounded at the free borders. They had large vesicular nuclei with a single nucleolus and a small amount of cytoplasm 
which was finely granular. The granules stained pale blue with haematoxylin and eosin, dark purple with Giemsa. Because of this modified lining of the air spaces it was often difficult to differentiate respiratory bronchioles from alveolar ducts or the latter from alveoli.

The whole interstitial tissue, and especially the inter-alveolar septa, were extremely infiltrated with numerous large mononuclear cells, lymphocytes, and many plasma cells (Fig. 3).

In sections stained by the Unna-Pappenheim method it was seen that many cells which had not the characteristic morphology of plasma cells had, like the latter, a strongly pyroninophil cytoplasm. No fibroblasts and no collagenous fibres were seen in the interalveolar septa. Although the interstitial cellular infiltration was diffuse it varied considerably in extent in various areas, which explains the patchy appearance on macroscopic examination.

The honeycombed material which filled the terminal air spaces was pale pinkish-violet in sections stained with haematoxylin and eosin, and in sections stained with Giemsa it was clearly seen to consist of closely packed rounded bodies, about $5 \mu$ in diameter, which showed a linear violet margin, a colourless area, a pale purplish central part, and one or two blackish-violet granules or short rods situated eccentrically or close to the margin (Figs. 3 and 4). This structure was equally well recognizable with Heidenhain's azan stain, when the outlines were purple, the granules dark purplish-red or bright red while the material which surrounded the granules stained pale sky-blue. Even better was the structure demonstrable by the McManus-Hotchkiss method, which has been recommended by Hamperl (1952) for this study. With this technique the outline and the granules were bright red, and often within a colourless area a bright red or pink spot was seen. With Masson's trichrome the material stained greenish-blue and with van Gieson's stain greyish-brown or yellowishbrown. Distinct pyroninophilia of granules, spots, and margins was seen in sections using UnnaPappenheim's stain. No fibrin was found in the material. By the coupled tetrazonium reaction of Danielli (1947), which demonstrates proteins by virtue of their contained tyrosine, tryptophane, and histidine, the honeycombed masses stained pale yellow, while the granules and short rods contrasted by their bright cherry-red colour. The same, though weaker, reaction was seen after benzoylation. It will be shown later that granules and rods represent protozoal chromatin and it is known that nuclear chromatin is comparatively refractory to benzoylation. This was originally attributed to the presence of purine- and pyrimidine-containing compounds. However, according to Pearse (1953) the coupled tetrazonium reaction is always due to the presence of the three previously mentioned aromatic amino-acids, and their relative resistance to benzoylation is the result of " physical" causes inherent in the spatial arrangement of the polypeptide chains of the proteins containing them making them susceptible to some kind of rearrangement through mild heat.

The interpretation of the positive P.A.S. reaction was somewhat difficult, probably because only material fixed with Orth's fluid was available. Not only was the plasmal reaction with $\mathrm{HgCl}_{2}$ oxidation positive, but also in the control preparation, in which untreated sections were placed in leucofuchsin, the material was stained bright red. This reaction was completely blocked by hydroxylamine, thus showing the presence of aldehydes, either in the original material or formed from acetalphosphatides by the action of the fixative which contains bichromate. It was therefore a surprise that prolonged ( 24 hours) acetylation with acetic anhydride in pyridine blocked completely the P.A.S. reaction. I would suggest that the explanation is to be found in a Canizaro reaction taking place during the prolonged acetylation of the material. Two molecules of pre-formed aldehyde yield one molecule of alcohol and one molecule of carboxylic acid. The former is then blocked by acetylation, the latter is intrinsically P.A.S. negative. It remained to decide whether 1-2 glycol groups were present in the honeycombed material in addition to pre-formed aldehydes. For this purpose the latter were blocked by hydroxylamine, a control section placed in leucofuchsin and another submitted to the P.A.S. reaction. The latter was strongly positive, and, all lipid stains being negative, it may be concluded that the material in addition to aldehydes also contained compounds with 1-2 glycol groups. As mentioned before, the granules and rods which gave a strong P.A.S. reaction represent protozoal chromatin. For comparison the P.A.S. reaction and acetylation were therefore performed on toxoplasma and the result was identical.

The material did not stain with mucicarmine. With Reinhart's modification of Hale's dialysediron method a patchy accumulation of acid mucopolysaccharides was demonstrable in some air spaces, but the actual honeycombed material did not stain. With the methylene blue-extinction method, according to Pearse (1953), granules and outlines were stained from $p \mathrm{H} 3.88$ upwards; the whole material was slightly stained at $p \mathrm{H} 5.32$ and 


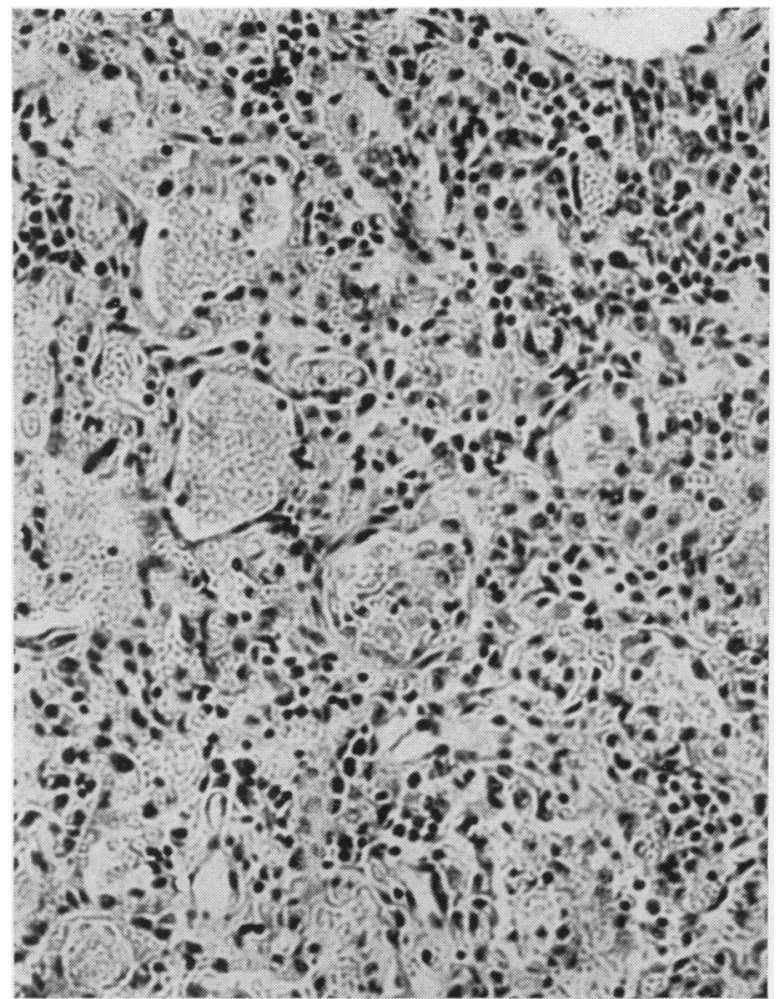

Fig. 1. - Honeycombed masses in the air spaces and infiltration of alveolar septa. Haematoxylin and cosin, $\times 150$.

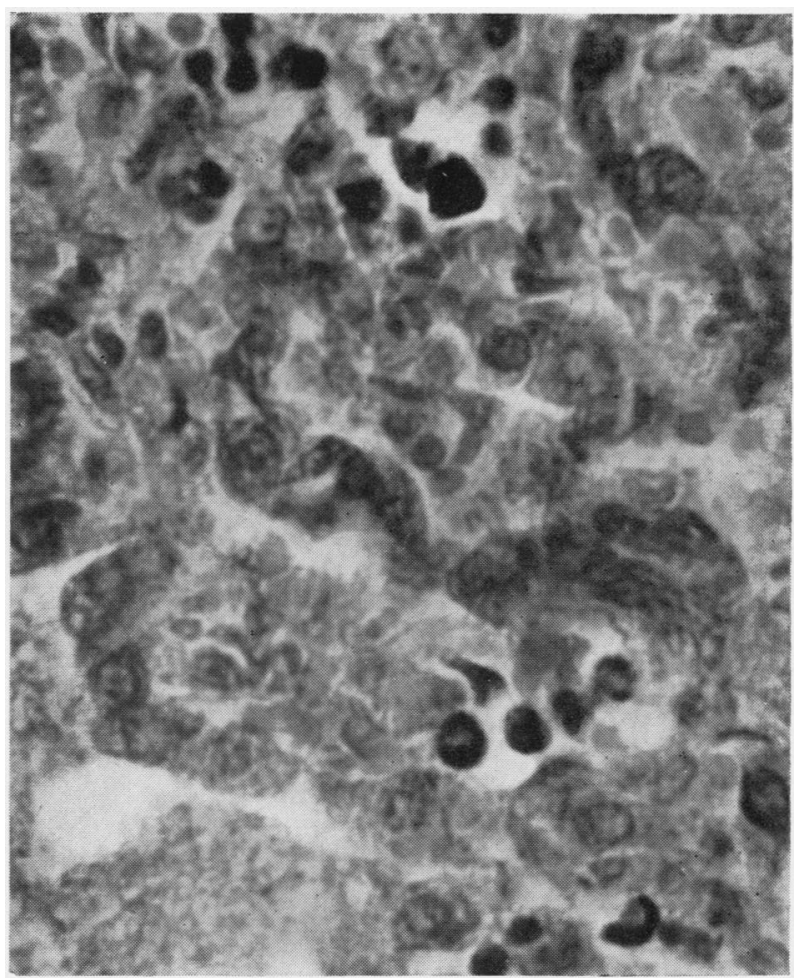

Fig. 2.-Epithelial lining of alveoli. LIn the left lower part typical honeycombed masses with protozoal chromatin granules are seen. Giemsa, $\times 625$.

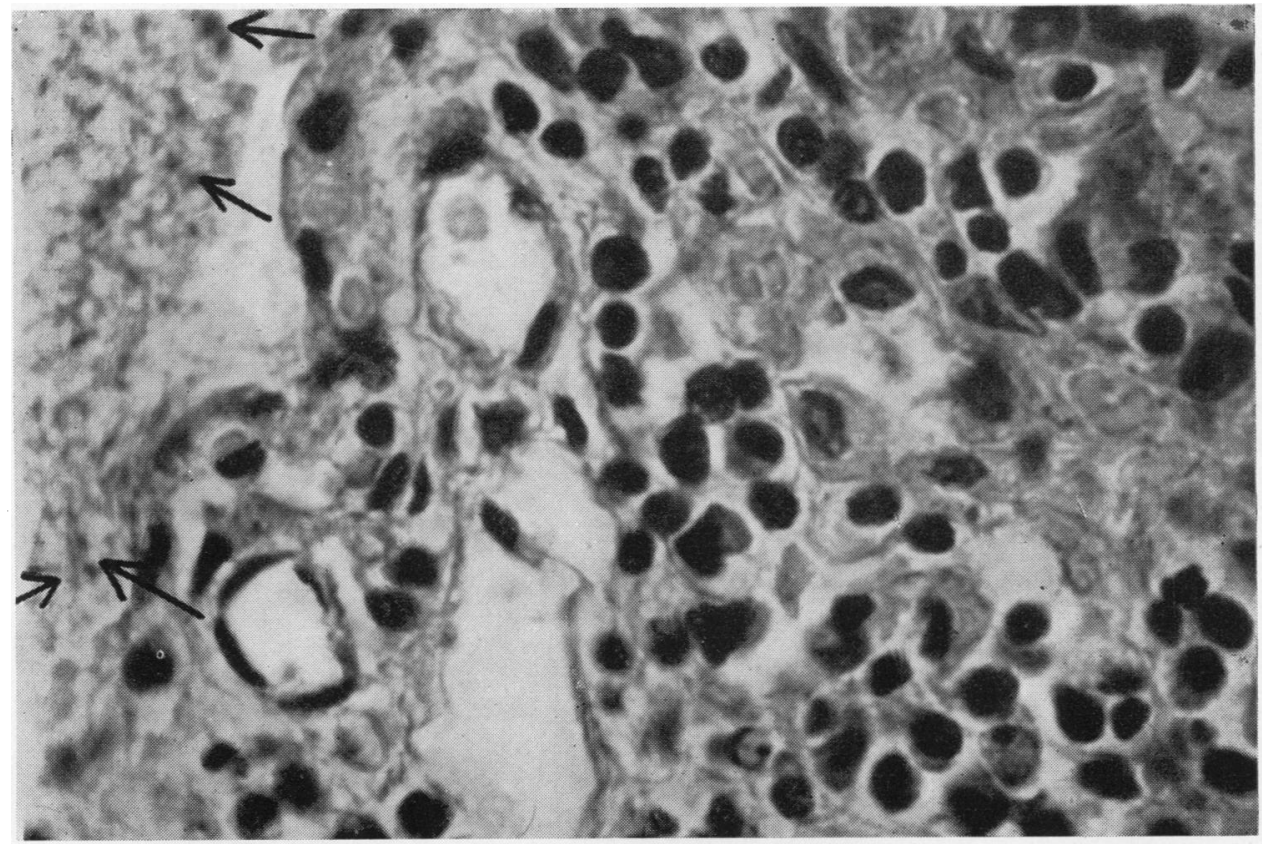

Fig. 3. - The cellular infiltration of interalveolar septa. The arrows on the left side point to protozoal chromatin in a honeycombed mass within an alveolus. Giemsa, $\times 625$. 


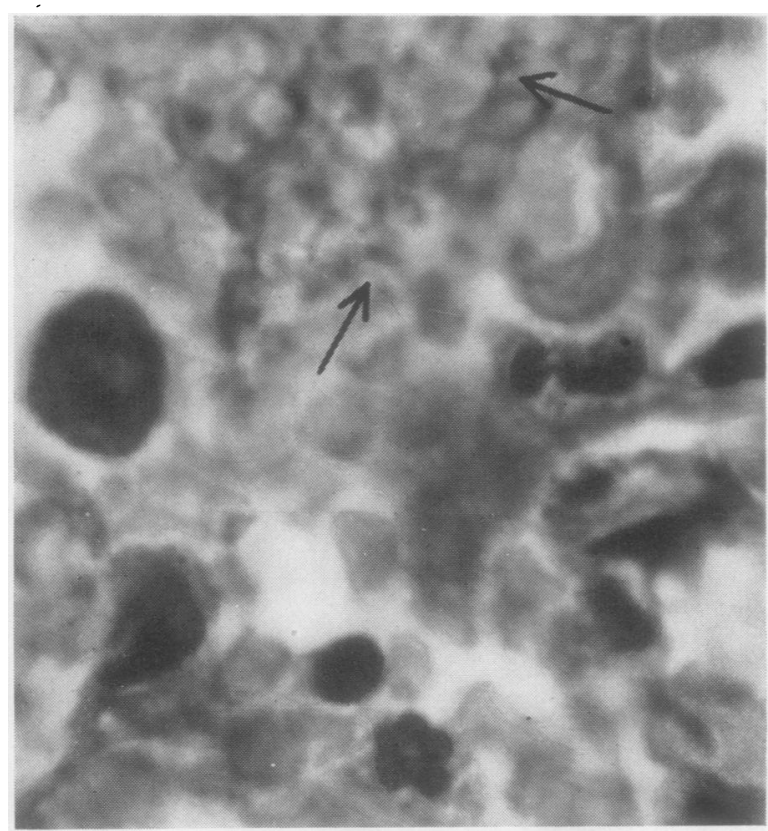

Fig. 4.-The arrows point to granules and rods of protozoal chromatin. Giemsa, $\times 1,350$.

distinctly from $p \mathrm{H} 6.99$ upwards. The positive stain at $p \mathrm{H} 3.88$ was, in view of the pyroninophilia, almost certainly due to ribonucleic acid. Treatment with ribonuclease $(1 \mathrm{mg}$. $/ \mathrm{ml}$. for two Hours at $37^{\circ} \mathrm{C}$.) completely abolished pyroninophilia and almost completely methylene blue, staining at a low $p H$. The same result was obtained by hydrolysis with $\mathrm{N} / 1 \mathrm{HCl}$ at $60^{\circ} \mathrm{C}$. for 10 minutes, according to Lajtha (1954). Only embedded material was available for investigation, and so only alcohol-fast metachromasia could be tested and this was negative.

Summarizing the results of staining and histochemical reactions it can be concluded that the honeycombed material consisted of globular bodies of hydropically swollen mucoproteins with circumscribed accumulations of ribonucleic acid. Within the mucoproteins were granules and rods of chromatin which contained large amounts of carbohydrate and ribonucleoproteins and in Orth-fixed material aldehydes which were either pre-formed or resulted from bichromate oxidation of acetalphosphatides.

This description of histological and histochemical findings shows clearly that plasmacellular interstitial pneumonia is entirely different from other forms of interstitial pneumonia of infants, such as the syphilitic pneumonia alba, Hecht's giant-cell pneumonia, and the more common monocytic interstitial pneumonia.
Apart from the pulmonary changes the only $\stackrel{2}{\Rightarrow}$ histological finding of interest was the presence $\stackrel{\vec{S}}{\rightarrow}$ of typical cytomegalic inclusions in the duct-cells? of the parotid and submaxillary glands (Fig. 5). 흘 No inclusions were found in the bronchial epi- $\frac{\bar{D}}{\vec{D}}$ thelium or in any other organ, none of which $\stackrel{\unrhd}{\varrho}$ showed any significant changes.

\section{Discussion}

Although many authors have in the past re- $\vec{\omega}$ marked on the peculiar honeycombed material in the air spaces in interstitial plasmacellular pneumonia, it was usually considered to be coagulated oedema fluid with cellular debris, and no further $\overrightarrow{0}$ attention was paid to this feature until Vaněk and 0 Jírovec in 1952 elucidated the nature of this material by the study of fresh lung-imprints. $T$ Characteristic bodies were found which on $\frac{\mathbb{P}}{0}$ morphological grounds were identified as the protozoon Pneumocystis carinii. This organism wase disovered by Chagas in the lungs of guinea-pigs $\vec{\bullet}$ which were infected with Trypanosoma cruzi. ज Chagas believed it to be a stage in the life cycle of trypanosoma, but later authors, such as Carinio and Maciel (1915) and Delanoë (1912), showed that it is unrelated to the trypanosoma cycle. Wenyon (1926) places it in the class of sporozoa, $\frac{\mathscr{Q}}{\mathbb{Q}}$ subclass coccidiomorpha. He states: "They un- $\frac{\varnothing}{2}$ doubtedly represent the schizogony stage of some $\overrightarrow{\overrightarrow{0}}$ sporozoon, and Stevenson suggests they may be schizonts of Klosiella." This place in the system of protozoa would make pneumocystis related to toxoplasma. Vaněk and Jírovec (1952) classify thes organism as a primitive haplosporidium, a well-i known representative of which species is the rhinosporidium.

All attempts up to the present time to culture the organism have been unsuccessful and so have animal inoculations. Vaněk and Jírovec's findings were confirmed by Hamperl (1952), by Herzberg? Herzberg-Kremmer, and May (1952), by Bachmann. (1953), and partly by Stopka (1952). Comparisono of our histological sections with photomicrographs of lung-imprints reproduced in the publications of Vaněk and Jírovec and of Herzberg et al. leaves scarcely any doubt, that the honeycombed materiak in the air-spaces represents masses of the protozo? which were identified by the Czech authors. If ins addition it is borne in mind how characteristic and different from any type of interstitial pneu monia is the histopathology of interstitial plasma cellular pneumonia, the assumption that Pneumo $\frac{\text { ? }}{\mathbb{P}}$ cystis carinii is the causative organism appear@ reasonable although only one of the three postu? lates of Robert Koch has been fulfilled. Hamper $\phi$ 
(1952) found the typical honeycombed structures in all seven cases which he examined. Herzberg et al. (1952) reported that the only case in which they failed to find pneumocystis in lung-imprints presented a different histopathology. Bachmann (1953) found pneumocystis in all of 14 cases of plasmacellular pneumonia which he examined. interstitial plasmacellular pneumonia have been reported up to the present time ; it is also common in this country, where the present case is the first one of interstitial plasmacellular pneumonia to be reported. Cytomegalic inclusion disease is as a rule congenital, transplacentally transmitted; recently we found a generalized cytomegalic inclu-

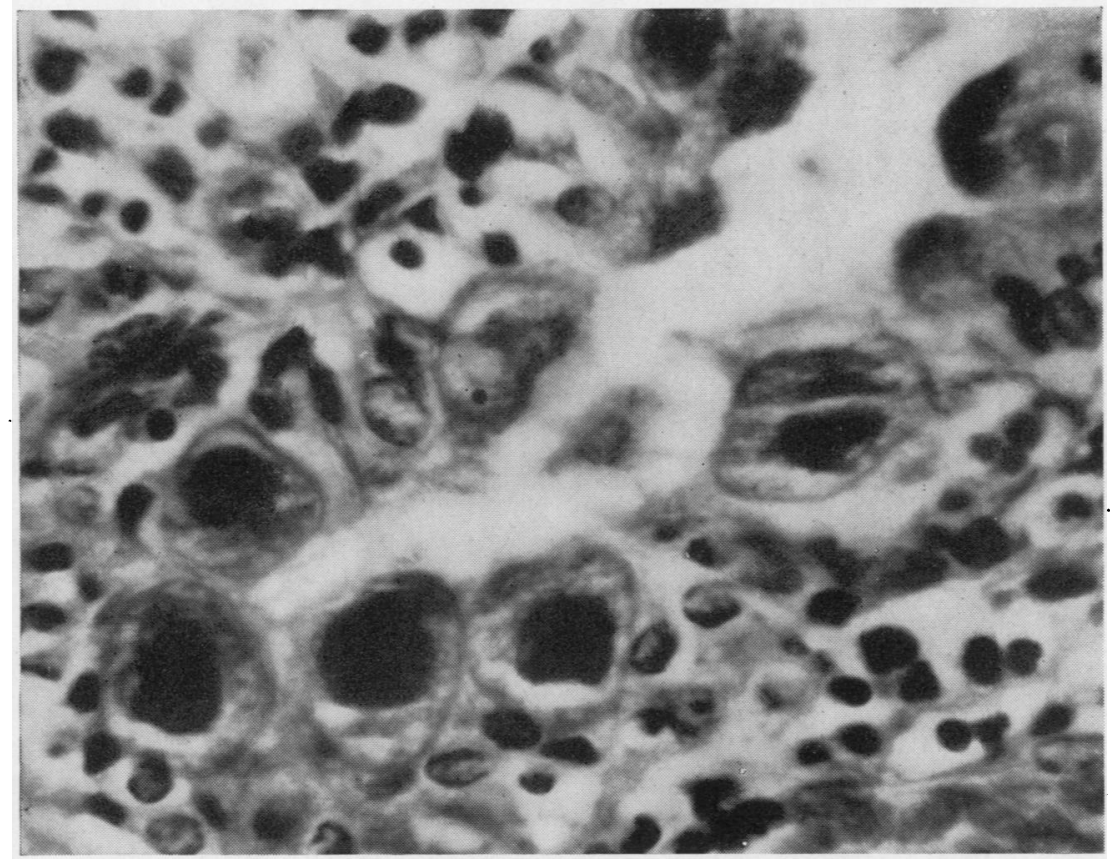

FIG. 5.-Cytomegalic inclusions in duct cells of parotid gland. Giemsa, $\times 650$.

Stopka's report (1952) that out of 17 cases typical foam structures were found only in eight and occasional suspicious appearances in two is probably due to confusion of plasmacellular with other forms of interstitial pneumonia. No histological findings were reported by this author. The same author, in a recent examination of five acute cases by the imprint method, found pneumocystis in all.

An interesting finding in our case is the association with cytomegalic inclusions in salivary glands. Since such inclusions were found in about $5 \%$ of the post-mortem material of Birmingham Children's Hospital it was at first considered that the association was coincidental. Recently, however, Dittrich and Seifert (1953) reported this association in 20 examples of interstitial plasmacellular pneumonia. Such a frequency rules out a chance association. For the understanding of the relationship of these two findings the following facts appear to be significant. Cytomegalic inclusion disease is common in the U.S.A., where no examples of sion disease in a macerated foetus. On the other hand, all features of interstitial plasmacellular pneumonia indicate a post-natal infection. No interstitial plasmacellular pneumonia was observed in any of the fatal cases of generalized cytomegalic inclusion disease, nor were cytomegalic inclusions seen in the bronchial epithelium of our case or of any other example of interstitial plasmacellular pneumonia. It is therefore clear that the two conditions are due to different causes. Cytomegalic inclusion disease of salivary glands is present before infection with Pneumocystis carinii takes place, and the reasonable exp'anation appears to be that the symptomless cytomegalic disease which is limited to the salivary glands predisposes to manifest interstitial plasmacellular pneumonia after infection with Pneumocystis carinii in a similar way as prematurity and marasmus do. Without such a predisposing factor the pneumocystis infection may remain symptomless. Thus the protozoon was frequently found in a variety of apparently 
healthy laboratory animals, in a man who died of Chagas' disease, in a 3-month-old infant with congenital heart disease, in a 4-month-old baby, and a 21-year-old man who died of unrelated disease (van der Meer and Brug, 1942).

In view of considerable evidence in favour of Pneumocystis carinii being the causative organism of interstitial plasmacellular pneumonia, the name parasitic pneumonia suggested by Vaněk and Jírovec appears justified.

The geographic distribution of the disease is puzzling, because the occurrence of Pneumocystis carinii in animals has been reported from various countries, and in Great Britain it was found by two authors (Coles, 1914 ; Porter, 1916).

The diagnosis is made from clinical symptoms and radiological findings. Pneumocystis has not yet been found in retropharyngeal swabs, but further investigations in this direction are advisable because the infection is resistant to chloromycetin, aureomycin, and other antibiotics, while it appears to respond favourably to simultaneous treatment with " plasmochin" and "atebrin" (Dvořák and Jírovec, 1953).

\section{Summary}

A case of interstitial plasmacellular pneumonia is described - the first to be reported outside the European continent. The finding of characteristic honeycombed masses in the air spaces, which were identified by Vaněk and Jírovec as the protozoon Pneumocystis carinii, is confirmed. Histochemical reactions of the honeycombed masses are reported The case was associated with cytomegalic incluos sions of salivary glands, and the significance ot this association is discussed.

This case was under the care of Dr. Frances Braid I wish to thank her for the clinical notes. Thanks are also due to Mr. A. R. Detheridge for technical assis tance and to Mr. J. G. Williamson for the photo: micrographs.

\section{REFERENCES}

Ammich, O. (1938). Virchows Arch. path. Anat., 302, 539.

Bachmann, K. D. (1953). Z. Kinderheilk., 73, 632.

Benecke, E. (1938). Verh. dtsch. path. Ges., 31, 402

Carini, A., and Maciel, J. (1915). Zbl. Bakt. I. Abt., 77, 46.

Coles, A. C. (1914). Parasitology, 7, 17.

Danielli, J. F. (1947). Nucleic Acid Symp. Soc exp Biol 1. 101.

Delanoë, P. (1912). C.R. Acad. Sci., Paris, 155, 658.

Dittrich, J. K., and Seifert, G. (1953). Z. Kinderheilk., 73, 639.

Dvoŕák, J., and Jírovec, O. (1953). Acta paediat., Uppsala, 42. 2:2Feyrter, F. (1927). Frankfurt Z. Path., 35, 213.

- (1941). Zbl. inn. Med., 62, 1.

Gloggengiesser, W. (1951). Frankfurt Z. Path., 62, 213.

Gormsen, H. (1950). Acta paediat., Uppsala, 39, 291.

Hamperl, H. (1952). Klin. Wschr., 30, 820. Herzberg, K., Herzberg-Kremmer, H., and May, G. (1952). Ibid
30, 822.

Lajtha, L. G. (1954). Nature, Lond., 173, 587.

Linell, F. (1949). Nord. Med., 41, 760.

Loeschcke, H. (1928). Verh. dtsch. path. Ges., 23, 291. 22, 301 .

Nitschke, A. (1940). Z. Kinderheilk. 62, 200.

Pearse, A. G. E. (1953). Histochemistry. Churchill, London.

Porter, A. (1916). Parasitology, 8, 255.

Rössle, R. (1923). Verh. dtsch. path. Ges., 19, 18.

(1928). Ibid., 23, 289.

Roulet, F. (1941). Schweiz. med. Wschr., 22, 1313.

Stopka, E. (1952). Kinderärztl. Prax., 20, 529.

Vaněk, J., and Jírovec, O. (1952). Zbl. Bakt. I. Abt. 158, 120

Weisse, K. (1951). Ergebn. inn. Med. Kinderheilk., n.s. 2, 611. Wenyon, C. M. (1926). Protozoology, Vol. 2. Ballière, Tindal
and Cox, London. 